\title{
Algebraic structure and integration maps in cocycle models for differential cohomology
}

\author{
MARKUS UPMEIER
}

\begin{abstract}
We construct explicit multiplicative and additive structures as well as integration maps on differential extensions of rationally even cohomology theories in the HopkinsSinger cocycle model. To this end, we consider also a pair-theory for which a long exact sequence is established.
\end{abstract}

$55 \mathrm{~N} 20 ; 55 \mathrm{~S} 05$

\section{Introduction}

Let $E$ be a multiplicative cohomology theory satisfying Milnor's wedge axiom. Then one has the multiplicative Chern-Dold transformation [8]

$$
\text { ch: } E^{n}(X, A) \rightarrow H^{n}(X, A ; V)=\prod_{i \in \mathbb{Z}} H^{i}\left(X, A ; V^{n-i}\right)
$$

of cohomology theories for the graded coefficient vector space $V^{*}=\pi_{-*} E \otimes \mathbb{R}$.

We always assume $E$ to be rationally even, meaning $V^{2 n+1}=0$ for all $n \in \mathbb{Z}$.

Definition 1.1 A differential extension [4] of $E$ consists of a contravariant functor $\widehat{E}$ from the category of manifolds to graded abelian groups along with natural linear transformations $I, R, a$ as in the following commutative diagram, which is required to have an exact upper horizontal row.

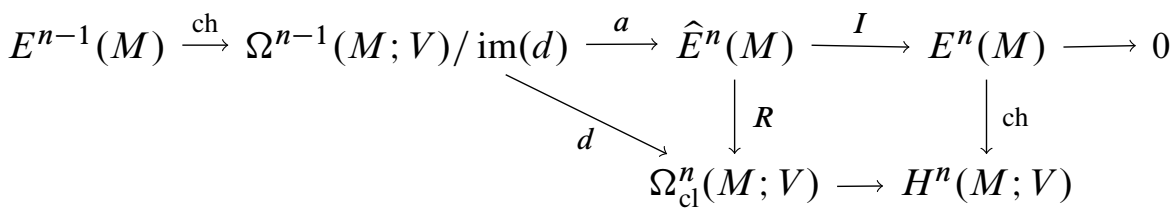

Definition 1.2 A multiplicative structure on $\widehat{E}$ consists of a unit $1 \in \widehat{E}^{0}(\mathrm{pt})$ and natural bilinear transformations

$$
\times: \widehat{E}^{n}(N) \times \widehat{E}^{m}(M) \rightarrow \widehat{E}^{n+m}(N \times M),
$$


which are associative, graded commutative, and unital. The maps $I, R$ are required to preserve the external product and unit, while for $a$ we demand

$$
a(\theta) \times x=a(\theta \bar{\wedge} R(x)) \text { for all } \theta \in \Omega^{n-1}(N ; V), x \in \hat{E}^{m}(M) .
$$

(Here, $\omega \bar{\wedge} \eta=\operatorname{pr}_{1}^{*} \omega \wedge \operatorname{pr}_{2}^{*} \eta$ denotes the external product of differential forms.)

Early examples of differential cohomology appeared as the sheaf-theoretic Deligne cohomology (see Gajer [10]) and Cheeger-Simons differential characters [6]. These provide a natural setting to study secondary invariants that take additional geometric structure into account. Later, a stratifold model for ordinary differential cohomology was introduced by Bunke, Kreck, and Schick [2]. Differential extensions of $K$-theory were studied by Lott [12] and Bunke and Schick [3] with which a refinement of the families index theorem may be proven; see Freed and Lott [9]. Another broad class of differential extensions (for Landweber exact cohomology theories) was constructed by Bunke, Schick, Schröder and Wiethaup [5].

Each of these examples carries a multiplicative structure. On the other hand, a general homotopy-theoretic construction of differential extensions was given by Hopkins and Singer [11], but its multiplicative properties remained unclear.

Their study is the main subject of this paper: Theorem 2.5 proves the existence of products for rationally even cohomology theories. As a second main result (Theorem 2.3) we construct explicit integration maps, which are crucial to extend constructions from even degrees to odd degrees. To this end, we introduce differential cohomology for pairs and exhibit a corresponding pair sequence in Theorem 2.2. Finally, in order to prove bilinearity of our products we exhibit a cocycle-based description of addition (Theorem 2.1), which is needed since the abelian group structure in [11] is given by producing a spectrum with homotopy groups $\widehat{E}^{n}(M)$ and the structure maps (in particular, the addition) are only abstractly given, involving choices of functorial sections [11, Equation (4.41)]. In summary, our results provide an accessible approach to (rationally even) differential cohomology based on concrete cocycle constructions.

\section{A Conventions and notation}

1A1 Spectrum By Brown's representability theorem, the reduced $E$-cohomology $\widetilde{E}^{n}(X)$ is represented by a spectrum $\left(E_{n}, \varepsilon_{n}: E_{n} \wedge S^{1} \rightarrow E_{n+1}\right)$ via pointed homotopy classes $\left[X, E_{n}\right]$ with homeomorphisms as adjoint structure maps $\varepsilon_{n}^{\text {adj: }} E_{n} \rightarrow \Omega E_{n+1}$. We fix such a choice of spectrum. 
$1 \mathrm{A2}$ Coefficients Let $C^{n}(X, A ; V)=\prod_{i+j=n} C^{i}\left(X, A ; V^{j}\right)$ denote cochains with coefficients in $V$ and similarly for cohomology and differential forms. Hence an $n$-cochain is a chain map $C_{*}(X, A) \rightarrow V_{*-n}$, where $V_{*}=V^{-*}$ is a chain complex with zero differential. By $C_{\mathrm{s}}^{*}(M, N ; V)$ we mean the subcomplex of smooth cochains. Relative differential forms $\omega \in \Omega^{n}(M, N ; V)$ (meaning $\omega_{p}=0$ at each $p \in N$ ) yield smooth cochains via the de Rham homomorphism.

1 A3 Integration Let $u \in C^{n+1}\left((X, A) \times S^{1} ; V\right)$. Using the Eilenberg-Zilber map EZ: $C_{*}(X, A) \otimes C_{*}\left(S^{1}\right) \rightarrow C_{*}\left((X, A) \times S^{1}\right)$ (see tom Diek [7, page 240]) the integral $\int u$ is the element of $C^{n}(X, A ; V)$ defined on chains $\sigma$ by $\left(\int u\right)(\sigma)=u\left(\operatorname{EZ}\left(\sigma \otimes S^{1}\right)\right)$, for the canonical $1-$ cycle $S^{1}$ on the circle. EZ is a natural chain map, hence

$$
\int \delta u=\delta \int u, \quad f^{*} \int u=\int\left(f \times \mathrm{id}_{S^{1}}\right)^{*} u .
$$

(i) The integral of $\omega \in \Omega^{n+1}\left(M \times S^{1} ; V\right)$ along the fiber $S^{1}$ is also denoted $\int \omega$. Relative forms are integrated by viewing them as absolute forms. In particular, $\int j^{*} \omega=\int \omega$ for $j:\left(M \times S^{1}, M \times 1\right) \rightarrow M \times S^{1}$.

(ii) For $c:(X, A) \times\left(S^{1}, 1\right) \rightarrow\left(E_{n+1}\right.$, pt) we let $\int c:(X, A) \rightarrow\left(E_{n}, \mathrm{pt}\right)$ denote the unique map $\left(\varepsilon_{n}^{\text {adj }}\right)^{-1} \circ c^{\text {adj }}$ with

$$
\varepsilon_{n} \circ\left(\int c \times \mathrm{id}_{S^{1}}\right)=c .
$$

(iii) In cohomology, (ii) induces $\int: E^{n+1}\left((X, A) \times\left(S^{1}, 1\right)\right) \rightarrow E^{n}(X, A)$ and an absolute integration map $\int: E^{n+1}\left(X \times S^{1}\right) \rightarrow E^{n}(X)$ (see Bunke and Schick [4, page 5]).

Combining (5) and (6), integration of cochains and of maps, as in (ii), are compatible:

$$
\int c^{*} u_{n}=\left(\int c\right)^{*} u_{n-1} \text { if } u_{n-1}=\int \varepsilon_{n-1}^{*} u_{n} .
$$

Viewing forms as cochains, cochain integration extends (i); see Hopkins and Singer [11, Lemma 3.15].

$1 \mathrm{~A} 4$ Left integration Suspensions are on the right, homotopies have their interval $I=[0,1]$ on the left. Let $\int^{\prime} u=u(\mathrm{EZ}(I \otimes-))$ denote the left integral of the cochain $u \in C^{n+1}(I \times(X, A) ; V)$ over the canonical $1-$ chain on $I$. Then

$$
\int^{\prime} \delta u+\delta \int^{\prime} u=\left.u\right|_{1 \times X}-\left.u\right|_{0 \times X}, \quad f^{*}\left(\int^{\prime} u\right)=\int^{\prime}\left(\operatorname{id}_{I} \times f\right)^{*} u .
$$

Let $\mathrm{pr}_{i j \ldots}$ be the projection $X_{1} \times X_{2} \times \cdots \rightarrow X_{i} \times X_{j} \times \cdots$ on the indicated factors. 


\section{Main results}

We briefly recall the Hopkins-Singer construction of differential cohomology: Following [11, page 48], fix a spectrum $\left(E_{n}, \varepsilon_{n}\right)$ representing $E$ and a choice of fundamental cocycles $\iota_{n} \in \widetilde{Z}^{n}\left(E_{n} ; V\right)$ satisfying

$$
\iota_{n-1}=\int \varepsilon_{n-1}^{*} \iota_{n} .
$$

These are representatives of the reduced cohomology classes $\left[\iota_{n}\right] \in \widetilde{H}^{n}\left(E_{n} ; V\right)$ that represent the reduced Chern character $(A=\mathrm{pt})$ via

$$
\operatorname{ch}[f]=f^{*}\left[\iota_{n}\right], \quad[f] \in\left[X, E_{n}\right] \cong \widetilde{E}^{n}(X) .
$$

Now, for $N \subset M$ closed, define $\widehat{E}^{n}(M, N)$ as the set of all equivalence classes of differential cocycles

$$
c:(M, N) \rightarrow\left(E_{n}, \mathrm{pt}\right)
$$

satisfying

$$
\delta h=\omega-c^{*} \iota_{n} \quad \text { for } \omega \in \Omega^{n}(M, N ; V), h \in C_{\mathrm{s}}^{n-1}(M, N ; V) .
$$

Here an equivalence $\left(c_{0}, \omega, h_{0}\right) \sim\left(c_{1}, \omega, h_{1}\right)$ is a pair

$$
C: I \times(M, N) \rightarrow\left(E_{n}, \mathrm{pt}\right), \quad H \in C_{\mathrm{s}}^{n-1}(I \times(M, N) ; V),
$$

restricting to $\left(c_{0}, h_{0}\right),\left(c_{1}, h_{1}\right)$ on the boundary, satisfying $\delta H=\mathrm{pr}^{*} \omega-C^{*} \iota_{n}$. To a smooth map of pairs $f:\left(M_{1}, N_{1}\right) \rightarrow\left(M_{2}, N_{2}\right)$ the functor $\widehat{E}$ associates

$$
\text { (11) } \hat{E}^{n}(f)=f^{*}: \hat{E}^{n}\left(M_{2}, N_{2}\right) \rightarrow \widehat{E}^{n}\left(M_{1}, N_{1}\right), \quad[c, \omega, h] \mapsto\left[c \circ f, f^{*} \omega, f^{*} h\right] \text {, }
$$

where [ ] denotes equivalence classes. Defining $I[c, \omega, h]=[c], R[c, \omega, h]=\omega$, $a(\theta)=$ [const, $d \theta, \theta]$ it is shown in [11, Equation (4.57)] that these groups form a differential extension of $E$ and that the associated flat theory $\hat{E}_{\text {flat }}^{n}(M)=\operatorname{ker}(R) \subset$ $\widehat{E}^{n}(M)$ is a cohomology theory.

In the framework of Hopkins-Singer differential cohomology we obtain the following results (always under the assumption rationally even).

Theorem 2.1 There is an explicit abelian group structure on $\widehat{E}^{n}(M, N)$ (described in Section 3A) for which $I, R, a$ and the induced maps (11) are linear.

The proof is given in Section 3B. In Section 4 and Section 5 we then prove Theorem 2.2 and Theorem 2.5, respectively. 
Theorem 2.2 For closed submanifolds $N \subset M$ we have an exact sequence:

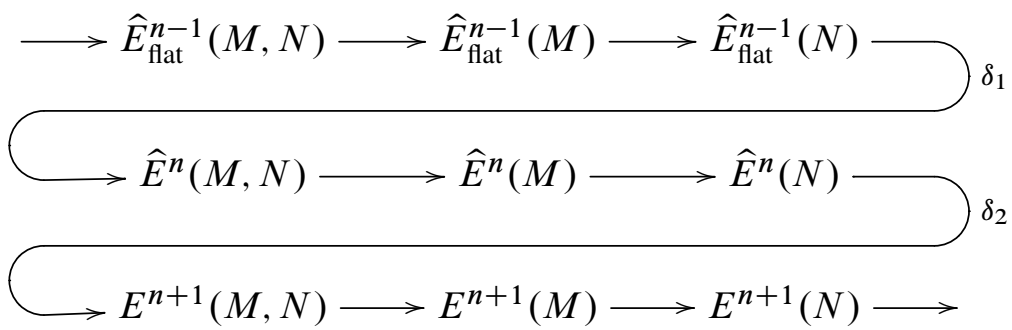

Here $\delta_{1}: \widehat{E}_{\text {flat }}^{n-1}(N) \rightarrow \widehat{E}_{\text {flat }}^{n}(M, N) \subset \widehat{E}^{n}(M, N)$ and $\delta_{2}: \widehat{E}^{n}(N) \stackrel{I}{\rightarrow} E^{n}(N) \rightarrow$ $E^{n+1}(M, N)$, using the connecting homomorphisms for $\widehat{E}_{\text {flat }}$ and $E$.

Theorem 2.3 The maps from Section 1A3 define linear natural transformations

$$
\int: \widehat{E}^{n+1}\left((M, N) \times\left(S^{1}, 1\right)\right) \rightarrow \widehat{E}^{n}(M, N), \quad[c, \omega, h] \mapsto\left[\int c, \int \omega, \int h\right] .
$$

These commute with $I, R, a$ (using (i) and (iii) from Section $1 A$ on forms and Ecohomology).

The main work is carried out in Section 6B where we prove our main result.

Theorem 2.4 There exists a multiplicative structure in even degrees. This product structure is compatible with integration maps ( $n, m$ even).

$$
\iint(x \times y)=x \times\left(\iint y\right) \text { for all } x \in \widehat{E}^{n}(N), y \in \widehat{E}^{m}\left(M \times S^{1} \times S^{1}\right)
$$

A formal argument in Section 6C then yields the following.

Theorem 2.5 A rationally even multiplicative cohomology theory $E$ admits a multiplicative differential extension with integration satisfying

$$
\int(x \times y)=x \times\left(\int y\right) \text { for all } x \in \widehat{E}^{n}(N), y \in \widehat{E}^{m}\left(M \times S^{1}\right) .
$$

\section{Addition}

Combining the universal coefficient and Künneth theorem with [4, Lemma 3.8] gives $\widetilde{H}^{k}\left(E_{n} \times E_{m} \times \cdots ; V\right)=0$ for $k$ odd and $n, m, \ldots$ even. Hence

$$
\delta x=0 \text { for } x \in \widetilde{C}^{k}\left(E_{n} \times E_{m} \times \cdots ; V\right) \Longrightarrow x \in \operatorname{im}(\delta) .
$$


We denote by $\varepsilon_{n}^{E \times E \times \cdots}$ the product spectra $E \times E \times \cdots$ structure maps

$$
\begin{aligned}
\left(E_{n} \times E_{n} \times \cdots\right) & \wedge S^{1} \rightarrow E_{n+1} \times E_{n+1} \times \cdots, \\
\left(x_{1}, x_{2}, \ldots\right) & \wedge t \mapsto\left(\varepsilon_{n}\left(x_{1} \wedge t\right), \varepsilon_{n}\left(x_{2} \wedge t\right), \ldots\right) .
\end{aligned}
$$

\section{A Construction}

In $E$-cohomology, addition is represented (using adjoint structure maps to write $E_{i} \approx \Omega^{2} E_{i+2}$ ) by loop composition $\alpha_{i}: E_{i} \times E_{i} \rightarrow E_{i}$ and negation by loop inversion $v_{i}: E_{i} \rightarrow E_{i}$ (using either loop coordinate). We agree to use the first or second coordinate for $\alpha_{i}, v_{i}$, according to whether $i$ is even or odd. Hence for even $n$,

$$
\alpha_{n-1}=\int \alpha_{n} \circ \varepsilon_{n-1}, \quad v_{n-1}=\int v_{n} \circ \varepsilon_{n-1} .
$$

Linearity of (1) implies $\operatorname{ch}\left[\mathrm{pr}_{1}\right]+\operatorname{ch}\left[\mathrm{pr}_{2}\right]=\operatorname{ch}\left[\alpha_{n}\right]$ and $-\operatorname{ch}[\mathrm{id}]=\operatorname{ch}\left[v_{n}\right]$. In terms of the fundamental cocycles, this means that we may pick (we do this only for even $n$ ) cochains $A_{n} \in \widetilde{C}^{n-1}\left(E_{n} \times E_{n} ; V\right), N_{n} \in \widetilde{C}^{n-1}\left(E_{n} ; V\right)$ with

$$
\delta A_{n}=\operatorname{pr}_{1}^{*} \iota_{n}+\operatorname{pr}_{2}^{*} \iota_{n}-\alpha_{n}^{*} \iota_{n}, \quad \delta N_{n}=-\iota_{n}-v_{n}^{*} \iota_{n} .
$$

To ensure (17) also in odd degrees, in view of (5), (7), (9), and (16) we set

$$
A_{n-1}=\int \varepsilon_{n-1}^{*} A_{n}, \quad N_{n-1}=\int \varepsilon_{n-1}^{*} N_{n} .
$$

Definition 3.1 The sum of differential cocycles is defined by

$$
\left(c_{1}, \omega_{1}, h_{1}\right)+\left(c_{2}, \omega_{2}, h_{2}\right)=\left(\alpha_{i}\left(c_{1}, c_{2}\right), \omega_{1}+\omega_{2}, h_{1}+h_{2}+\left(c_{1}, c_{2}\right)^{*} A_{i}\right) .
$$

Define also $0=($ const $, 0,0)$ and $-(c, \omega, h)=\left(v_{i} c,-\omega,-h+c^{*} N_{i}\right)$. These are all differential cocycles by (17) and since the fundamental cocycles are reduced.

\section{B Verification of axioms}

With Definition 3.1, induced maps (11) and $I, R, a$ are clearly linear. To check that (19) descends to differential cohomology, suppose $\left(C_{1}, H_{1}\right): x_{1} \sim x_{1}^{\prime},\left(C_{2}, H_{2}\right): x_{2} \sim x_{2}^{\prime}$ are equivalences. Then

$$
\left(\alpha_{i}\left(C_{1}, C_{2}\right), H_{0}+H_{1}+\left(C_{0}, C_{1}\right)^{*} A_{i}\right): x_{1}+x_{2} \sim x_{1}^{\prime}+x_{2}^{\prime} .
$$


The following two rules for manipulating differential cocycles are proven in the appendix (as Lemmas A.1 and A.2):

$$
\begin{aligned}
& h-h^{\prime} \in \operatorname{im}(\delta) \Longrightarrow[c, \omega, h]=\left[c, \omega, h^{\prime}\right] \\
& C: c_{0} \simeq c_{1}(\operatorname{rel} N) \Longrightarrow\left[c_{0}, \omega, h\right]=\left[c_{1}, \omega, h-\int^{\prime} C^{*} \iota_{n}\right] \in \widehat{E}^{n}(M, N) .
\end{aligned}
$$

Remark 3.2 For $n$ even, any two choices of $A_{n}$ or $N_{n}$ differ by a cocycle, which is a coboundary by (15). Combining (5) with (18), this is true also in odd degrees. Hence (20) implies that the addition in differential cohomology depends only on the spectrum $\left(E_{n}, \varepsilon_{n}\right)$ and the choice of fundamental cocycles.

Lemma 3.3 Suppose $H: I \times E_{n} \times E_{m} \times \cdots \rightarrow E_{i}$ is a pointed homotopy and $c$ is a $\operatorname{map}(M, N) \rightarrow\left(E_{n} \times E_{m} \times \cdots, \mathrm{pt}\right)$. Then $x=\left[H_{0} \circ c, \omega, h\right], y=\left[H_{1} \circ c, \omega, h^{\prime}\right]$ in $\widehat{E}^{i}(M, N)$ agree if there exists $v_{i} \in \widetilde{Z}^{i-1}\left(E_{n} \times E_{m} \times \cdots ; V\right)$ with

$$
h-h^{\prime}-c^{*} \int^{\prime} H^{*} \iota_{i} \equiv c^{*} v_{i} \bmod \operatorname{im}(\delta),
$$

and one of the following conditions is satisfied:

(i) $i$ and $n, m, \ldots$ are even integers.

(ii) $i=n=m=\cdots$ are odd and there exists $v_{i+1} \in \widetilde{Z}^{i}\left(E_{i+1} \times E_{i+1} \times \cdots ; V\right)$ with $v_{i}=\int\left(\varepsilon_{i}^{E \times E \times \cdots}\right)^{*} v_{i+1}$.

Proof Given (i), $\delta v_{i}=0$ and (15) shows that $v_{i}$ is a coboundary. If (ii) is satisfied, then $v_{i+1}$ is a coboundary by (i) and (5) implies that $v_{i}=\int \varepsilon_{i}^{*} v_{i+1}$ is also a coboundary. In each case, the cochain (22) is a coboundary. Hence

$$
\begin{aligned}
{\left[H_{0} \circ c, \omega, h\right] } & =\left[H_{1} \circ c, \omega, h-c^{*} \int^{\prime} H^{*} \iota_{i}\right] & \text { by }(8),(21) \\
& =\left[H_{1} \circ c, \omega, h^{\prime}\right] & \text { by }(20) .
\end{aligned}
$$

Theorem 2.1 There is an explicit abelian group structure on $\widehat{E}^{n}(M, N)$ (described in Section 3A) for which $I, R, a$ and induced maps (11) are linear.

Proof Expressing that $E$-cohomology is abelian group-valued in terms of representing maps leads to homotopies (chosen only for even degrees $n$ )

$$
\begin{aligned}
H_{n}^{\text {ass }}: \alpha_{n}\left(\alpha_{n} \times \mathrm{id}\right) & \simeq \alpha_{n}\left(\mathrm{id} \times \alpha_{n}\right), & H_{n}^{\text {neg }}: \alpha_{n}\left(\mathrm{id}, v_{n}\right) \simeq \text { const }, \\
H_{n}^{\text {zer }}: \alpha_{n}(\mathrm{id}, \mathrm{const}) & \simeq \mathrm{id}, & H_{n}^{\text {com }}: \alpha_{n} \circ \text { flip } \simeq \alpha_{n} .
\end{aligned}
$$


In view of (16), corresponding homotopies in degree $n-1$ may be defined by

$$
\begin{aligned}
& H_{n-1}^{\text {ass }}=\int H_{n}^{\text {ass }} \circ\left(\operatorname{id}_{I} \times \varepsilon_{n-1}^{E \times E \times E}\right), \quad H_{n-1}^{\text {neg }}=\int H_{n}^{\text {neg }} \circ\left(\operatorname{id}_{I} \times \varepsilon_{n-1}\right),
\end{aligned}
$$

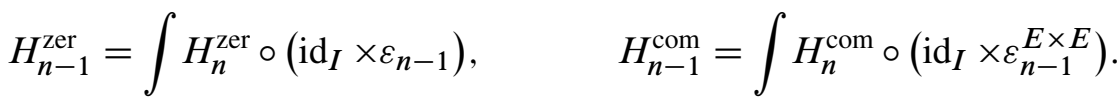

For $x_{j}=\left[c_{j}, \omega_{j}, h_{j}\right]$ in $\widehat{E}^{i}(M, N), j=1,2,3$, we must show

$$
\left(x_{1}+x_{2}\right)+x_{3}=x_{1}+\left(x_{2}+x_{3}\right), \quad x_{1}+\left(-x_{1}\right)=0, \quad x_{1}+0=x_{1}, \quad x_{2}+x_{1}=x_{1}+x_{2} .
$$

Unwinding Definition 3.1 leads in each case to differential cocycles $x, y$ we wish to prove are equivalent; eg unwinding the commutativity axiom leads to

$$
\begin{aligned}
& x=\left(\alpha_{i}\left(c_{2}, c_{1}\right), \omega_{2}+\omega_{1}, h_{2}+h_{1}+\left(c_{2}, c_{1}\right)^{*} A_{k}\right), \\
& y=\left(\alpha_{i}\left(c_{1}, c_{2}\right), \omega_{1}+\omega_{2}, h_{1}+h_{2}+\left(c_{1}, c_{2}\right)^{*} A_{k}\right) .
\end{aligned}
$$

Each of these axioms holds for differential forms and is exhibited in differential cohomology by a respective application of Lemma 3.3 to the cochains

$$
\begin{aligned}
v_{k}^{\mathrm{ass}} & =\operatorname{pr}_{12}^{*} A_{k}+\left(\alpha_{k} \times \mathrm{id}\right)^{*} A_{k}-\operatorname{pr}_{23}^{*} A_{k}-\int^{\prime}\left(H_{k}^{\mathrm{ass}}\right)^{*} \iota_{k}, \\
v_{k}^{\mathrm{com}} & =\mathrm{flip}^{*} A_{k}-A_{k}-\int^{\prime}\left(H_{k}^{\mathrm{com}}\right)^{*} \iota_{k}, \\
v_{k}^{\text {neg }} & =N_{k}+\left(\mathrm{id}, v_{k}\right)^{*} A_{k}-\int^{\prime}\left(H_{k}^{\mathrm{neg}}\right)^{*} \iota_{k}, \\
v_{k}^{\text {zer }} & =(\mathrm{id}, \text { const })^{*} A_{k}-\int^{\prime}\left(H_{k}^{\text {zer }}\right)^{*} \iota_{k},
\end{aligned}
$$

the maps $c^{\text {ass }}=\left(c_{1}, c_{2}, c_{3}\right), c^{\text {com }}=c^{\text {neg }}=\left(c_{1}, c_{2}\right), c^{\text {zer }}=c_{1}$, and homotopies $H=$ $H_{i}^{\text {ass }}, H_{i}^{\text {com }}, H_{i}^{\text {neg }}, H_{i}^{\text {zer }}$. Our choice (17) and (8) ensure $\delta v_{k}=0$ in each case. If $i$ is odd, condition (ii) of Lemma 3.3 holds by (9), (18), and (23).

Assuming the coefficients $E^{*}(\mathrm{pt})$ are countably generated, [4, Theorem 3.10] implies that $\hat{E}^{*}$, with the above abelian group structure and the integration maps from Section 5, is isomorphic to the construction in [11].

\section{Long exact sequence of pairs}

Proof of Theorem 2.2 At every place except $\hat{E}^{n}(M)$ the exactness follows by combining the exactness of (2) with the exact pair sequences for $E, \widehat{E}_{\text {flat }}$. Suppose therefore that $(c, \omega, h)$ is a cocycle on $M$ with an equivalence $(C, H)$ from $\left.(c, \omega, h)\right|_{N}$ 
to (const, 0,0). Let $\phi: I \rightarrow I$ be smooth strictly increasing with $\left.\phi\right|_{[0,1 / 4]}=0, \phi(1)=1$. Pulling $C, H$ back along $\phi$, we may suppose

$$
\left.C\right|_{[0,1 / 4] \times N}=\left.c\right|_{N} \circ \operatorname{pr}_{2},\left.\quad H\right|_{[0,1 / 4] \times N}=\left.\operatorname{pr}_{2}^{*} h\right|_{N}
$$

Applying Lemma A.3 to $A=0 \times M \cup[0,1 / 4[\times N, B=] 0,1] \times N$ and $\left.\operatorname{pr}_{2}^{*} h\right|_{A}, H$, we find $G \in C_{\mathrm{s}}^{n-1}(0 \times M \cup I \times N ; V)$ with $\left.G\right|_{A}=\left.\operatorname{pr}_{2}^{*} h\right|_{A},\left.G\right|_{B}=H$.

The maps $c$ and $C$ can be glued to a map $D$ on $0 \times M \cup I \times N$. In view of (35), cocycles are invariant under sd. Hence, to prove $\delta G=\operatorname{pr}^{*} \omega-D^{*} \iota_{n}$, it suffices to consider chains contained entirely in $A$ or in $B$, which reduces to the corresponding facts for $h$ and $H$. Since $N \subset M$ is a cofibration, we find a retraction $r: I \times M \rightarrow 0 \times M \cup I \times N$. Deforming $r$ relative to the closed subset $M \times 0 \cup I \times N$, we may suppose that $r$ is smooth. The pullback of $(G, D)$ along $r$ satisfies $\delta r^{*} G=\operatorname{pr}^{*} \omega-(D r)^{*} \iota_{n}$ and therefore represents an equivalence $(c, \omega, h) \sim\left(\left.D \circ r\right|_{M \times 1}, \omega,\left.r^{*} G\right|_{M \times 1}\right)$ to an element of $\widehat{E}^{n}(M, N)$.

\section{Integration maps}

Combining (5), (7), and (9) shows that $\left(\int c, \int \omega, \int h\right)$ from (13) is indeed a differential cocycle. If $(C, H)$ is an equivalence $x_{0} \sim x_{1}$, then we have $\delta \int H=\operatorname{pr}^{*} \omega-\left(\int C\right)^{*} \iota_{n}$ and so $\int x_{0} \sim \int x_{1}$. Hence (13) is well defined.

Theorem 2.3 The maps from Section 1A3 define linear natural transformations

$$
\int: \hat{E}^{n+1}\left((M, N) \times\left(S^{1}, 1\right)\right) \rightarrow \hat{E}^{n}(M, N), \quad[c, \omega, h] \mapsto\left[\int c, \int \omega, \int h\right] .
$$

These commute with $I, R, a$ (using (i) and (iii) from Section $1 A$ on forms and $E-$ cohomology).

Proof Naturality for smooth $f: M_{1} \rightarrow M_{2}$ means

$$
\widehat{E}^{n}(f)\left(\int[c, \omega, h]\right)=\int \widehat{E}^{n+1}\left(f \times \mathrm{id}_{S^{1}}\right)[c, \omega, h],
$$

and follows from (5). Linearity of (13) asserts an equality

$$
\begin{aligned}
\int & {\left[\alpha_{i}\left(c_{1}, c_{2}\right), \omega_{1}+\omega_{2}, h_{1}+h_{2}+\left(c_{1}, c_{2}\right)^{*} A_{i}\right] } \\
= & {\left[\alpha_{i-1}\left(\int c_{1}, \int c_{2}\right), \int \omega_{1}+\int \omega_{2}, \int h_{1}+\int h_{2}+\left(\int c_{1}, \int c_{2}\right)^{*} A_{i-1}\right] . }
\end{aligned}
$$


If $i=n$ is even, (7), (18) imply $\int\left(c_{1}, c_{2}\right)^{*} A_{n}=\left(\int c_{1}, \int c_{2}\right)^{*} A_{n-1}$ and by (16) we have $\int \alpha_{i}\left(c_{1}, c_{2}\right)=\alpha_{i-1}\left(\int c_{1}, \int c_{2}\right)$, so (24) holds. Addition in $E$-cohomology may be performed using either loop coordinate, so we may select a homotopy

$$
H: \alpha_{i-1} \circ\left(\varepsilon_{i-1}^{\mathrm{adj}} \times \varepsilon_{i-1}^{\mathrm{adj}}\right)^{-1} \simeq\left(\varepsilon_{i-1}^{\mathrm{adj}}\right)^{-1} \circ \Omega \alpha_{i} .
$$

If $i=n-1$ is odd, applying (21) with $H \circ\left(c_{1}^{\mathrm{adj}}, c_{2}^{\mathrm{adj}}\right)$ reduces us by (20) to showing that the following is a coboundary (which follows from (15)):

$$
A_{i}-\left(\varepsilon_{i-1}^{\mathrm{adj}} \times \varepsilon_{i-1}^{\mathrm{adj}}\right)^{*} \int^{\prime} H^{*} \iota_{i}+\int \varepsilon_{i}^{*} A_{i+1} .
$$

Corollary 5.1 From (13) we obtain unique linear natural transformations

$$
\int: \widehat{E}^{n+1}\left(M \times S^{1}\right) \rightarrow \widehat{E}^{n}(M)
$$

satisfying the following two conditions:

(i) The map (13) is the composition of (25) with $\widehat{E}^{n+1}(j)$.

(ii) $\int \mathrm{pr}_{1}^{*}=0$ for the projection $\mathrm{pr}_{1}: M \times S^{1} \rightarrow M$.

Here, $j: M \times S^{1} \rightarrow M \times\left(S^{1}, 1\right)$. Moreover, (25) commutes with $I, R, a$.

Proof Write $i: M \times 1 \rightarrow M \times S^{1}$ and let $x \in \widehat{E}^{n+1}\left(M \times S^{1}\right)$. By exactness of (12), we have $x-\operatorname{pr}_{1}^{*} i^{*} x=j^{*} y$ for some class $y \in \widehat{E}^{n+1}\left(M \times S^{1}, M \times 1\right)$. Then

$$
\int x \stackrel{(\mathrm{ii})}{=} \int\left(x-\mathrm{pr}_{1}^{*} i^{*} x\right) \stackrel{(\mathrm{i})}{=} \int y
$$

shows uniqueness and gives a formula for existence. To check that (26) is well defined, assume $j^{*} y=0$. Since $i$ is a section of $\mathrm{pr}_{1}$, the exact sequence of pairs in $E-$ cohomology implies that $E^{n+1}(j)$ is injective, so $I(y)=0$. By (2), write $y=a(\theta)$. Since $0=j^{*} y=a\left(j^{*} \theta\right)$, write $j^{*} \theta=\operatorname{ch}(t)$ for some $t \in E^{n}\left(M \times S^{1}\right)$. Using that (1) is compatible with suspension, we have

$$
\int y=\int a(\theta)=a\left(\int \theta\right)=a\left(\int j^{*} \theta\right)=a\left(\int \operatorname{ch}(t)\right)=a\left(\operatorname{ch}\left(\int t\right)\right)=0,
$$

where the second equality follows from Theorem 2.3 , and the last from (2). This proves well definedness. Linearity and compatibility of (25) with $I, R, a$ is inherited from the corresponding properties in Theorem 2.3. 


\section{A Integration from the left}

Define left integration $\int^{\prime}=\int \tau^{*}$ using the flip $\tau$ of the two circles in $M \times S^{1} \times S^{1}$. Lemma $5.2 \iint \tau^{*}=-\iint: \widehat{E}^{n+2}\left(M \times\left(S^{1}, 1\right) \times\left(S^{1}, 1\right)\right) \rightarrow \widehat{E}^{n}(M) \quad(n$ even $)$.

Proof The respective assertion is true for differential forms (graded commutativity), cochains (by the symmetry properties of EZ), and maps (if $t: E_{n} \approx \Omega^{2} E_{n+2} \rightarrow$ $\Omega^{2} E_{n+2} \approx E_{n}$ flips the two loop coordinates, we have $\left.t \circ \iint c=\iint c \circ \tau\right)$. Hence, selecting a homotopy $H: v_{n} \simeq t$, we may apply Lemma 3.3(i) with $v_{k}=\int^{\prime} H^{*} \iota_{k}-$ $\left(v_{k}\right)^{*} N_{k}, H, \iint c$, to prove equality of

$$
x=\left[t \iint c, \iint \tau^{*} \omega, \iint \tau^{*} h\right], \quad y=\left[v_{n} \iint c,-\iint \omega,-\iint h+\left(\iint c\right)^{*} N_{n}\right],
$$

provided we have $\delta v_{k}=0$, which follows from (8), (17), and the observation

$$
\begin{aligned}
t^{*} \iota_{k} & =t^{*} \iint\left(\varepsilon_{k} \times \mathrm{id}_{S^{1}}\right)^{*} \varepsilon_{k+1}^{*} \iota_{k+2}=\iint \tau^{*}\left(\varepsilon_{k} \times \mathrm{id}_{S^{1}}\right)^{*} \varepsilon_{k+1}^{*} \iota_{k+2} \\
& =-\iint\left(\varepsilon_{k} \times \mathrm{id}_{S^{1}}\right)^{*} \varepsilon_{k+1}^{*} \iota_{k+2}=-\iota_{k},
\end{aligned}
$$

where we have used (9) and the assertion $\iint=-\iint \tau^{*}$ for cochains.

Corollary $5.3 \iint \tau^{*}=-\iint: \widehat{E}^{n+2}\left(M \times S^{1} \times S^{1}\right) \rightarrow \widehat{E}^{n}(M) \quad(n$ even $)$.

\section{Products}

\section{A Construction in even degrees}

The unit and the multiplication in $E$-cohomology may be represented by pointed maps

$$
\mu_{n m}: E_{n} \wedge E_{m} \rightarrow E_{n+m}, \quad u: \mathrm{pt} \rightarrow E_{0} .
$$

Since (1) is multiplicative, $\operatorname{ch}\left[\operatorname{id}_{E_{n}}\right] \times \operatorname{ch}\left[\operatorname{id}_{E_{m}}\right]=\operatorname{ch}\left[\operatorname{id}_{E_{n+m}}\right], \operatorname{ch}[u]=1$. Hence we find cochains $M_{n, m} \in \widetilde{C}^{n+m-1}\left(E_{n} \wedge E_{m} ; V\right)$ and $U \in \widetilde{C}^{-1}(\mathrm{pt} ; V)$ with

$$
\delta M_{n, m}=\iota_{n} \times \iota_{m}-\iota_{n+m}, \quad \delta U=\omega_{p t}-u^{*} \iota_{0},
$$

where $\omega_{\mathrm{pt}}=1 \in \Omega^{0}(\mathrm{pt})$. The definition of corepresentable functor on $\mathcal{M}$ is rather technical, so we will not recall it here (see [1] or [13, Section 7]); an example is the smooth cochain functor $(M, N, \ldots) \mapsto C_{\mathrm{s}}^{k}(M \times N \times \cdots ; W)$ on manifolds with corners $\mathcal{C}=\operatorname{Man} \times \operatorname{Man} \times \cdots$ with models $\left(\Delta^{n}, \Delta^{m}, \cdots\right)$. 
Theorem (Acyclic models) Let $F, G$ be contravariant functors from a category $\mathcal{C}$ equipped with a full subcategory of models $\mathcal{M}$ to non-negative real cochain complexes. Suppose $G$ is corepresentable with respect to the models and that $H^{*+1}(F(M))=0$ for all $M \in \mathcal{M}$. Then any two natural chain maps $f^{*}, g^{*}: F^{*} \rightarrow G^{*}$ with $H^{0}(f)=$ $H^{0}(g)$ are naturally chain homotopic. Moreover, any two natural chain homotopies between natural chain maps are naturally chain homotopic.

Taking $F(M, N)=\Omega\left(M ; V^{i}\right) \otimes \Omega\left(N ; V^{j}\right), G(M, N)=C_{\mathrm{s}}^{*}\left(M \times N ; V^{i} \otimes V^{j}\right)$ and the chain maps $\wedge$ and $\cup$ gives natural chain homotopies $B_{i j}$ satisfying $\delta B_{i j}\left(\omega_{1} \otimes \omega_{2}\right)+B_{i j} d\left(\omega_{1} \otimes \omega_{2}\right)=\omega_{1} \bar{\wedge} \omega_{2}-\omega_{1} \times \omega_{2}, \quad \omega_{1} \in \Omega\left(M ; V^{i}\right), \omega_{2} \in \Omega\left(N ; V^{j}\right)$. Combining these as $B\left(\sum_{i} \omega^{i} \otimes \sum_{j} \omega^{j}\right)=\sum_{i, j} B_{i j}\left(\omega^{i} \otimes \omega^{j}\right)$, we get a natural chain homotopy $B$ for the case of graded coefficients satisfying

$$
\delta B\left(\omega_{1}, \omega_{2}\right)+B d\left(\omega_{1} \otimes \omega_{2}\right)=\omega_{1} \bar{\wedge} \omega_{2}-\omega_{1} \times \omega_{2} .
$$

Similarly, using $F(M, N)=C_{\mathrm{s}}^{*}(N \times M ; V), G$ above, and the chain maps $\times$ and $\tau^{*} \times$, the acyclic models theorem proves that the product of cochains is graded commutative up to natural chain homotopy $D$

$$
\delta D(u \otimes v)+D \delta(u \otimes v)=u \times v-(-1)^{|u||v|} \tau^{*}(v \times u) .
$$

Since the external product $\bar{\lambda}$ is graded commutative, both $(-1)^{\left|\omega_{0}\right|\left|\omega_{1}\right|} \tau^{*} B\left(\omega_{0}, \omega_{1}\right)-$ $D\left(\omega_{1}, \omega_{0}\right)$ and $B\left(\omega_{1}, \omega_{0}\right)$ define natural chain homotopies between

$$
\Omega(M ; V) \otimes \Omega(N ; V) \rightarrow C_{\mathrm{s}}^{*}(N \times M ; V), \quad\left(\omega_{0}, \omega_{1}\right) \mapsto \omega_{1} \bar{\wedge} \omega_{0}, \omega_{1} \times \omega_{0},
$$

and are so themselves chain homotopic. In particular, for closed forms $\omega_{0}, \omega_{1}$

$$
B\left(\omega_{1}, \omega_{0}\right) \equiv(-1)^{\left|\omega_{0}\right|\left|\omega_{1}\right|} \tau^{*} B\left(\omega_{0}, \omega_{1}\right)-D\left(\omega_{1}, \omega_{0}\right) \bmod \operatorname{im}(\delta) .
$$

Since $B\left(\omega_{\mathrm{pt}},-\right)$ and 0 are both natural chain homotopies id $\simeq \mathrm{id}$, the acyclic models theorem implies that they are themselves chain homotopic. Hence

$$
B\left(\omega_{\mathrm{pt}}, \omega\right) \in \operatorname{im}(\delta) \text { for all forms } \omega \text { with } d \omega=0 .
$$

Definition 6.1 The unit $1 \in \widehat{E}^{0}(\mathrm{pt})$ is defined by $\left[u, \omega_{\mathrm{pt}}, U\right]$. The product $x_{1} \times x_{2}$ of the differential cocycles $x_{1}=\left(c_{1}, \omega_{1}, h_{1}\right), x_{2}=\left(c_{2}, \omega_{2}, h_{2}\right)$ is

$$
\left(\mu_{n m}\left(c_{1} \times c_{2}\right), \omega_{1} \bar{\wedge} \omega_{2}, B\left(\omega_{1}, \omega_{2}\right)+h_{1} \times \omega_{2}+\omega_{1} \times h_{2}-h_{1} \times \delta h_{2}+\left(c_{1} \times c_{2}\right)^{*} M_{n, m}\right) .
$$

Naturality of the products follows from the naturality of $B, \bar{\wedge}, \delta$, and $\times$. Clearly $R$ and $I$ preserve the external product. (28), (29) ensure that the unit and $x_{1} \times x_{2}$ are 
indeed differential cocycles. Suppose we have equivalences $\left(C_{1}, H_{1}\right): x_{1} \sim x_{1}^{\prime}$ and $\left(C_{2}, H_{2}\right): x_{2} \sim x_{2}^{\prime}$. Then $C=\mu_{n, m}\left(C_{1} \times C_{2}\right)$ and the cochain

$H=B\left(\operatorname{pr}^{*} \omega_{1} \otimes \mathrm{pr}^{*} \omega_{2}\right)+H_{1} \times \mathrm{pr}^{*} \omega_{2}+\mathrm{pr}^{*} \omega_{1} \times H_{2}-H_{1} \times \delta H_{2}+\left(C_{1} \times C_{2}\right)^{*} M_{n, m}$ show $x_{1} \times x_{2} \sim x_{1}^{\prime} \times x_{2}^{\prime}$. Hence the product descends to a map (3) on differential cohomology.

\section{B Verification of axioms}

Let $\pi(x, y, z)=(x, y, x, z)$.

Theorem 2.4 We have a multiplicative structure in even degrees. This product structure is compatible with integration maps (14).

Proof Let $x_{j}=\left[c_{j}, \omega_{j}, h_{j}\right]$ for classes $x_{1} \in \widehat{E}^{n}(N), x_{2} \in \widehat{E}^{m}(M), x_{3} \in \widehat{E}^{l}(L)$ and even integers $n, m, l$.

(i) Bilinearity Let $x=x_{1} \times\left(x_{2}+x_{3}\right), y=x_{1} \times x_{2}+x_{1} \times x_{3}$. Unwinding Definitions 3.1 and 6.1, we see that we must compare the maps

$\mu_{n m}\left(\operatorname{id} \times \alpha_{m}\right) \circ c, \quad \alpha_{n+m} \circ\left(\mu_{n m} \times \mu_{n m}\right) \circ \pi \circ c$, where $c=c_{1} \times c_{2} \times c_{3}$,

differential forms $\omega=\omega_{1} \bar{\wedge}\left(\omega_{2}+\omega_{3}\right)=\omega^{\prime}=\omega_{1} \bar{\wedge} \omega_{2}+\omega_{1} \bar{\wedge} \omega_{3}$, and cochains

$$
\begin{gathered}
h=B\left(\omega_{1}, \omega_{2}+\omega_{3}\right)+h_{1} \times\left(\omega_{2}+\omega_{3}\right)+\omega_{1} \times\left(h_{2}+h_{3}+\left(c_{2}, c_{3}\right)^{*} A_{m}\right) \\
-h_{1} \times \delta\left(h_{2}+h_{3}+\left(c_{2}, c_{3}\right)^{*} A_{m}\right)+\left(c_{1} \times \alpha\left(c_{2}, c_{3}\right)\right)^{*} M_{n m}, \\
h^{\prime}=B\left(\omega_{1}, \omega_{2}+\omega_{3}\right)+h_{1} \times \omega_{2}+h_{1} \times \omega_{3}+\omega_{1} \times\left(h_{2}+h_{3}\right)-h_{1} \times \delta\left(h_{2}+h_{3}\right) \\
+\left(c_{1} \times c_{2}\right)^{*} M_{n m}+\left(c_{1} \times c_{3}\right)^{*} M_{n m}+\left(\mu\left(c_{1} \times c_{2}\right), \mu\left(c_{1} \times c_{3}\right)\right)^{*} A_{n+m} .
\end{gathered}
$$

Expressing that the product in $E$-cohomology is bilinear leads to a homotopy

$$
H: \mu_{n m}\left(\mathrm{id} \times \alpha_{m}\right) \simeq \alpha_{n+m}\left(\mu_{n m} \times \mu_{n m}\right) \pi .
$$

Using bilinearity of $B$ and of $\times$ for cochains shows that $h-h^{\prime}-c^{*} \int^{\prime} H^{*} \iota_{n+m}$ is the pullback along $c$ of the cochain $v$ on $E_{n} \times E_{m} \times E_{m}$ given by

$\iota_{n} \times A_{m}+\left(\left(\mathrm{id} \times \alpha_{m}\right)^{*}-\mathrm{pr}_{12}^{*}-\mathrm{pr}_{13}^{*}\right) M_{n m}-\pi^{*}\left(\mu_{n m} \times \mu_{n m}\right)^{*} A_{n+m}-\int^{\prime} H^{*} \iota_{n+m}$.

The cochain $v$ is closed by (8), (17), and (28). We conclude $x=y$ by an application of Lemma 3.3(i) to $H, c$, and $v$. 
(ii) Commutativity We have $\omega_{1} \bar{\wedge} \omega_{2}=\tau^{*}\left(\omega_{2} \bar{\wedge} \omega_{1}\right)$. Combining (30), (31) with (20) shows that $y=\tau^{*}\left(x_{2} \times x_{1}\right)=\left[\mu_{m n}\left(c_{2}, c_{1}\right) \circ \tau, \omega_{1} \bar{\wedge} \omega_{2}, h^{\prime}\right]$, where $h^{\prime}=B\left(\omega_{1}, \omega_{2}\right)+h_{1} \times \omega_{2}+\omega_{1} \times h_{2}-h_{1} \times \delta h_{2}+\left(c_{2} \times c_{1}\right)^{*} \tau^{*} M_{m n}+\left(c_{2} \times c_{1}\right)^{*} D\left(\iota_{m}, \iota_{n}\right)$. Then $y=x_{1} \times x_{2}$ by Lemma 3.3(i), applied to a homotopy $H: \mu_{m n} \circ \tau \simeq \mu_{n m}$, $c=c_{1} \times c_{2}$, and the following closed (by (8), (28), and (30)) cochain:

$$
v=\tau^{*} D\left(\iota_{m}, \iota_{n}\right)+\tau^{*} M_{m n}-M_{n m}-\int^{\prime} H^{*} \iota_{n+m} .
$$

(iii) Unitality Equation (32) and Lemma 3.3(i) applied to $H: \mu_{0 n}(u \times$ id) $\simeq$ id, the cochain $v_{n}=U \times \iota_{n}+(u \times \mathrm{id})^{*} M_{0 n}-\int^{\prime} H^{*} \iota_{n}$, and $c=c_{1}$ show $1 \times x_{1}=x_{1}$.

(iv) Associativity This property follows from Lemma 3.3(i) applied to $c=c_{1} \times c_{2} \times c_{3}$, a homotopy $H: \mu_{n, m+l}\left(\mathrm{id} \times \mu_{m l}\right) \simeq \mu_{n+m, l}\left(\mu_{n m} \times \mathrm{id}\right)$, and $v$ given by

$$
M_{n, m+l}-\mu_{n, m+l}^{*}\left(\iota_{n} \times M_{m l}\right)-M_{n+m, l}-\mu_{n+m, l}^{*}\left(M_{n m} \times \iota_{l}\right)-\int^{\prime} H^{*} \iota_{n+m+l} .
$$

(v) Compatibility with $\boldsymbol{a}$ Let $\theta \in \Omega^{n-1}(N ; V)$. Applying (20) to $B\left(d \theta, \omega_{2}\right) \equiv$ $\theta \bar{\wedge} \omega_{2}-\theta \times \omega_{2}$ and $d \theta \times h \equiv \theta \times \delta h$ (Leibniz' rule) modulo coboundaries gives

$$
a(\theta) \times x_{2}=\left[\mu_{n m}\left(\text { const } \times c_{2}\right), d \theta \pi \omega_{2}, \theta \pi \omega_{2}+\left(\text { const } \times c_{2}\right)^{*} M_{n m}\right] .
$$

Then $a(\theta) \times x_{2}=a\left(\theta \bar{\wedge} \omega_{2}\right)$ by Lemma 3.3(i) applied to $c=$ const $\times c_{2}$, a homotopy $H: \mu_{n m}($ const, id $) \simeq$ const, and the cocycle

$$
v=(\text { const } \times \mathrm{id}){ }^{*} M_{n m}-\int^{\prime} H^{*} \iota_{n+m} .
$$

(vi) Compatibility with integration (14) Let $\left[c_{1}, \omega_{1}, h_{1}\right] \in \widehat{E}^{n}(N)$ and $\left[c_{2}, \omega_{2}, h_{2}\right] \in$ $\widehat{E}^{m}\left(M \times S^{1} \times S^{1}\right)$ for $n, m$ even. Unwinding the definitions, we see that we need to compare

$x=\left[\iint \mu\left(c_{1} \times c_{2}\right), \iint\left(\omega_{1} \bar{\wedge} \omega_{2}\right), h\right], \quad y=\left[\mu\left(c_{1} \times \iint c_{2}\right), \omega_{1} \bar{\wedge}\left(\iint \omega_{2}\right), h^{\prime}\right]$ for the cochains $h$ and $h^{\prime}$ given by

$$
\begin{gathered}
\iint\left(B\left(\omega_{1}, \omega_{2}\right)+\omega_{1} \times h_{2}+h_{1} \times \omega_{2}-h_{1} \times \delta h_{2}+\left(c_{1} \times c_{2}\right)^{*} M_{n, m}\right), \\
B\left(\omega_{1}, \iint \omega_{2}\right)+h_{1} \times\left(\iint \omega_{2}\right)+\omega_{1} \times\left(\iint h_{2}\right)-h_{1} \times \delta\left(\iint h_{2}\right)+\left(c_{1} \times \iint c_{2}\right)^{*} M_{n, m-2} .
\end{gathered}
$$

Both $\iint B(-,-)$ and $B\left(-, \iint-\right)$ define natural chain homotopies between the same chain maps. By acyclic models, they are themselves chain-homotopic and hence 
differ on closed forms only by coboundary. Integration of forms is compatible with $\bar{\lambda}$ and integration of cochains is compatible with $\times$ (since the Eilenberg-Zilber map is a section of the Alexander-Whitney map, which is used to define the product of cochains), so

$$
h-h^{\prime} \equiv \iint\left(c_{1} \times c_{2}\right)^{*} M_{n, m}-\left(c_{1} \times \iint c_{2}\right)^{*} M_{n, m-2} \quad \bmod \operatorname{im}(\delta) .
$$

Since products in $E$-cohomology are compatible with suspension we may select a homotopy $H: \mu_{n m}\left(\mathrm{id} \times \varepsilon_{m-1} \Sigma \varepsilon_{m-2}\right) \simeq \varepsilon_{n+m-1} \Sigma \varepsilon_{n+m-2} \Sigma^{2} \mu_{n, m-2}$. Applying Lemma 3.3(i) with $c=c_{1} \times \iint c_{2}, \iint H$, and the cochain

$$
v=\iint\left(\mathrm{id} \times \varepsilon_{m-1} \circ \Sigma \varepsilon_{m-2}\right)^{*} M_{n m}-M_{n, m-2}-\int^{\prime}\left(\iint H\right)^{*} \iota_{n+m-2}
$$

proves $x=y$. Here, $\delta v=0$ follows from (5), (8), and (28).

\section{C Extension to odd degrees}

Theorem 2.5 is obtained from Theorem 2.4 by the following general principle.

Theorem 6.2 Suppose $\widehat{E}$ is a differential extension of $E$ such that we have:

(i) Long exact sequences (12) for every closed submanifold $N \subset M$.

(ii) Integration maps as in Theorem 2.3. By (i), these induce an absolute integration (25) for which we assume Corollary 5.3.

(iii) A multiplicative structure on $\widehat{E}$ in even degrees satisfying (14).

Then there is a unique extension of the multiplicative structure to all degrees, compatible with integration:

$$
\int(x \times y)=x \times\left(\int y\right) \text { for all } x \in \widehat{E}^{n}(N), y \in \widehat{E}^{m}\left(M \times S^{1}\right) .
$$

Lemma 6.3 Integration $\int: \widehat{E}^{n+1}\left(M \times S^{1}, M \times 1\right) \rightarrow \widehat{E}^{n}(M)$ is surjective. The kernel consists of all $a(\theta), \theta \in \Omega_{\mathrm{cl}}^{n}\left(M \times S^{1}, M \times 1 ; V\right)$, with $\left[\int \theta\right] \in \mathrm{im}(\mathrm{ch})$.

Proof Let $x \in \widehat{E}^{n}(M)$. The isomorphism $\int: E^{n+1}\left(M \times S^{1}, M \times 1\right) \cong E^{n}(M)$ and the surjectivity of $I$ show that there is $X \in \widehat{E}^{n+1}\left(M \times S^{1}, M \times 1\right)$ with

$$
\int I(X)=I(x)
$$


Exactness of (2) implies $x-\int X=a(\theta)$ for $\theta \in \Omega^{n-1}(M ; V)$. Let $\alpha: S^{1} \rightarrow \mathbb{R}$ be smooth with $\int \alpha(t) d t=1, \alpha(1)=0$. Then the form $\kappa=\operatorname{pr}_{1}^{*} \theta \wedge \alpha(t) d t \in$ $\Omega^{n}\left(M \times S^{1}, M \times 1 ; V\right)$ satisfies $\int \kappa=\theta$. Hence $X+a(\kappa)$ is a preimage of $x$.

To compute the kernel, suppose $\int X=0$. Then $\int I(X)=0$ and the isomorphism above show $I(X)=0$. By exactness of (2), $X=a(\theta)$ for some $\theta$. Now $a\left(\int \theta\right)=\int X=0$ implies $\left[\int \theta\right] \in \operatorname{im}(\mathrm{ch})$.

According to the lemma, every $y \in \widehat{E}^{m}(M)$ equals $\int Y$ for some $Y \in \widehat{E}^{m}\left(M \times S^{1}\right)$. By (33), the product is uniquely determined in all degrees.

Definition 6.4 For $x \in \widehat{E}^{n}(N), y \in \widehat{E}^{m}(M)$ pick $X \in \widehat{E}^{n+1}\left(N \times S^{1}\right)$ and $Y \in$ $\widehat{E}^{m+1}\left(M \times S^{1}\right)$ with $\int X=x, \int Y=y$. Let

$$
x \times y= \begin{cases}\int(X \times y) & n \text { odd, } m \text { even, } \\ \int(x \times Y) & n \text { even, } m \text { odd, } \\ \int(x \times Y)=-\int(X \times y) & n \text { odd, } m \text { odd. }\end{cases}
$$

(The last equality is by Corollary 5.3).

Lemma 6.5 Equation (34) is well defined.

Proof Suppose, for example, that $\int X=x=\int X^{\prime}$ in the case $n$ odd, $m$ even. In the notation of Corollary 5.1, by (12) we find $Z \in \widehat{E}^{n+1}\left(N \times S^{1}, N \times 1\right)$ with

$$
j^{*} Z=\left(X-X^{\prime}\right)-\operatorname{pr}_{1}^{*} i^{*}\left(X-X^{\prime}\right) .
$$

Thus $\int Z=\int\left(X-X^{\prime}\right)=0$ and Lemma 6.3 shows that $Z=a(\theta)$ for some $\theta$ with $\left[\int \theta\right] \in \mathrm{im}(\mathrm{ch})$. By commutativity of (2), the transformation $R$ maps to forms which represent classes in the image of the Chern character (which is multiplicative). Hence $\left(\int \theta\right) \bar{\wedge} R y=\int\left(j^{*} \theta \bar{\wedge} R y\right) \in \mathrm{im}(\mathrm{ch})$. We conclude

$$
\int\left(X-X^{\prime}\right) \times y=\int\left(j^{*} Z\right) \times y=\int a\left(j^{*} \theta\right) \times y=\int a\left(j^{*} \theta \bar{\wedge} R y\right)=0,
$$

using Corollary 5.1(ii), $a \circ \mathrm{ch}=0$ from (2), and the compatibility of $\times$ with $a$ in even degrees. The argument in the other cases is similar.

Proof of Theorem 6.2 Considering the definition (34) case by case, we see that (14) implies (33). Combined with Lemma 6.3, the verification of bilinearity, unitality, associativity, and graded commutativity (note our choice of sign in (34)) may therefore be reduced to the even-degree case. It remains to check

$$
a(\theta) \times x=a(\theta \bar{\wedge} R x) \quad \text { for } \theta \in \Omega^{n-1}(N ; V), x \in \widehat{E}^{m}(M),
$$


in case $n$ or $m$ are odd. For $\theta \in \Omega^{n-1}(N ; V)$ we find $\kappa \in \Omega^{n}\left(N \times S^{1} ; V\right)$ with $\int \kappa=\theta$. The verification reduces to even degrees; eg for $n$ odd and $m$ even

$$
a(\theta) \times x=\int(a(\kappa) \times x)=\int a(\kappa \bar{\wedge} R x)=a\left(\int \kappa \bar{\wedge} R x\right)=a(\theta \bar{\wedge} R x) .
$$

\section{Appendix: Technical lemmas}

Lemma A.1 For every smooth cochain $v \in C_{\mathrm{s}}^{n-1}(M, N)$ there exists $E \in Z_{\mathrm{s}}^{n}(I \times$ $(M, N))$ with restrictions $\left.E\right|_{0}=0$ and $\left.E\right|_{1}=\delta v$. Hence, if $(c, \omega, h)$ is a differential cocycle and $h-h^{\prime}$ is a coboundary, we have $(c, \omega, h) \sim\left(c, \omega, h^{\prime}\right)$.

Proof For the zeroth vertex $e_{1} \in \Delta^{n}$ and $\sigma=\left(\sigma_{1}, \sigma_{2}\right): \Delta^{n} \rightarrow I \times M$ we define $E(\sigma)=\sigma_{1}\left(e_{1}\right) v\left(\partial \sigma_{2}\right)$. Since $\sigma_{1} d^{i}\left(e_{1}\right)$ is independent of $i, E$ is a cocycle:

$$
E(\partial \sigma)=\sum(-1)^{i}\left(\sigma_{1} d^{i}\right)\left(e_{1}\right) v\left(\partial\left(\sigma_{2} \circ d^{i}\right)\right)=\sigma_{1}\left(e_{1}\right) v\left(\partial \partial \sigma_{2}\right)=0 .
$$

Applying this to each factor, we deduce a version with graded coefficients. In (20) it suffices for $h-h^{\prime}$ to bound a singular cochain (since $H^{i}(M, N ; V) \cong H_{\mathrm{s}}^{i}(M, N ; V)$ it follows that $h-h^{\prime}$ also bounds a smooth cochain then).

Lemma A.2 For a homotopy $C: c_{0} \simeq c_{1}($ rel $N)$ and $\left[c_{0}, \omega, h\right] \in \widehat{E}^{n}(M, N)$ we have $\left[c_{0}, \omega, h\right] \sim\left[c_{1}, \omega, h^{\prime}\right]$ for $h^{\prime}=h-\int^{\prime} C^{*} \iota_{n}$.

Proof Define a homotopy $K: C \simeq c_{1} \circ \mathrm{pr}_{2}$ by $K(s, t, m)=C(t, m)$ for $s \leq t$ and $K(s, t, m)=C(s, m)$ for $s \geq t$ and let $H=\operatorname{pr}_{2}^{*} h^{\prime}+\int^{\prime} K^{*} \iota_{n}$. Using (8) and $\delta h^{\prime}=\omega-c_{1}^{*} \iota_{n}$, we have $\delta H=\operatorname{pr}_{2}^{*} \omega-C^{*} \iota_{n}$ and $\left.H\right|_{0}=h^{\prime}+\int^{\prime} C^{*} \iota_{n}=h$, so the pair $(C, H)$ shows $\left[c_{0}, \omega, h\right]=\left[c_{1}, \omega,\left.H\right|_{1}\right]$. Since $\int^{\prime} \operatorname{pr}_{2}^{*} \omega=0$ for forms, we get

$$
\left.H\right|_{1}=h^{\prime}+\int^{\prime} \operatorname{pr}_{2}^{*} c_{1}^{*} \iota_{n}=h^{\prime}+\int^{\prime} \operatorname{pr}_{2}^{*}\left(\omega-\delta h^{\prime}\right) \stackrel{(8)}{=} h^{\prime}-\delta \int^{\prime} \operatorname{pr}_{2}^{*} h^{\prime} .
$$

Hence (20) implies $\left[c_{1}, \omega,\left.H\right|_{1}\right]=\left[c_{1}, \omega, h^{\prime}\right]$.

Lemma A.3 Let $M=A \cup B$ for open $A, B \subset M$. Any $u \in C_{\mathrm{s}}^{n}(A ; V), v \in C_{\mathrm{s}}^{n}(B ; V)$ with common restriction to $A \cap B$ may be extended to $C_{\mathrm{s}}^{n}(A \cup B ; V)$.

Proof We begin with the ungraded case, so $V$ is just a vector space and $u, v$ are $V$-valued cochains. Define a subdivision operator on smooth $n$-chains by

$$
\operatorname{sd}\left(\sigma_{n+1}\right)=(-1)^{n} \partial \sigma+\sigma_{n+1}, \quad \sigma_{n+1}: \Delta^{n} \rightarrow M,
$$


where $\sigma=\sigma_{n+1} \circ \pi$ for $\pi\left(t_{0}, \ldots, t_{n+1}\right) \mapsto\left(t_{0}+\frac{t_{n+1}}{n+1}, \ldots, t_{n}+\frac{t_{n+1}}{n+1}\right)$. Since the diameters tend to zero, after a finite minimal number $m(\sigma)$ of subdivisions any simplex $\sigma$ becomes a chain $\sum n_{k} \tau_{k}$ with every $\tau_{k}$ entirely in $A$ or in $B$. Define a $V$-valued cochain $w$ by

$$
w(\sigma)=\sum n_{k} \begin{cases}u\left(\tau_{k}\right) & \text { if } \tau_{k}\left(\Delta^{n}\right) \subset A, \\ v\left(\tau_{k}\right) & \text { if } \tau_{k}\left(\Delta^{n}\right) \subset B .\end{cases}
$$

By the minimality assumption, $w$ restricts to $u$ and $v$. This concludes the proof in the ungraded case. For the graded case, suppose $u=\left(u^{i}\right)_{i \in \mathbb{Z}}$ and $v=\left(v^{i}\right)_{i \in \mathbb{Z}}$ where $u^{i} \in C_{\mathrm{s}}^{i}\left(A ; V^{n-i}\right)$ and $v^{i} \in C_{\mathrm{s}}^{i}\left(B ; V^{n-i}\right)$. By assumption, $u^{i}$ and $v^{i}$ agree on $A \cap B$, hence by the already proven case we find $w^{i} \in C_{\mathrm{s}}^{i}\left(A \cup B ; V^{n-i}\right)$, extending $u^{i}$ and $v^{i}$. The family $w=\left(w^{i}\right)_{i \in \mathbb{Z}}$ is then the sought-for cochain.

\section{References}

[1] M Barr, Acyclic models, CRM Monograph Series 17, Amer. Math. Soc. (2002) MR1909353

[2] U Bunke, M Kreck, T Schick, A geometric description of differential cohomology, Ann. Math. Blaise Pascal 17 (2010) 1-16 MR2674652

[3] U Bunke, T Schick, Smooth K-theory, Astérisque 328, Soc. Math. France, Paris (2009) 45-135 MR2664467

[4] U Bunke, T Schick, Uniqueness of smooth extensions of generalized cohomology theories, J. Topol. 3 (2010) 110-156 MR2608479

[5] U Bunke, T Schick, I Schröder, M Wiethaup, Landweber exact formal group laws and smooth cohomology theories, Algebr. Geom. Topol. 9 (2009) 1751-1790 MR2550094

[6] J Cheeger, J Simons, Differential characters and geometric invariants, from: "Geometry and topology", Lecture Notes in Math. 1167, Springer, Berlin (1985) 50-80 MRMR827262

[7] T tom Dieck, Algebraic topology, EMS Textbooks in Mathematics, Eur. Math. Soc., Zürich (2008) MR2456045

[8] A Dold, Relations between ordinary and extraordinary homology, from: "Colloquium on Algebraic Topology”, Matematisk Institut, Aarhus Universitet, Denmark (1962) 2-9 MR0146039

[9] DS Freed, J Lott, An index theorem in differential K-theory, Geom. Topol. 14 (2010) 903-966 MR2602854

[10] P Gajer, Geometry of Deligne cohomology, Invent. Math. 127 (1997) 155-207 MR1423029 
[11] M J Hopkins, I M Singer, Quadratic functions in geometry, topology, and M-theory, J. Differential Geom. 70 (2005) 329-452 MR2192936

[12] J Lott, $\mathbb{R} / \mathbb{Z}$ index theory, Comm. Anal. Geom. 2 (1994) 279-311 MR1312690

[13] M Upmeier, Algebraic structure and integration in generalized differential cohomology, $\mathrm{PhD}$ thesis, Georg-August-Universität Göttingen (2013) Available at http:// hdl . handle.net/11858/00-1735-0000-0022-5E03-9

Mathematisches Institut, Universität Augsburg Universitätsstrasse 14, 86159 Augsburg, Germany markus . upmeier@math . uni-augsburg. de

Received: 16 August 2012 Revised: 16 February 2014 
